ORIGINAL ARTICLE

\title{
Does a single bolus thrombolytic reduce door to needle time in a district general hospital?
}

\author{
V Leah, C Clark, K Doyle, T J Coats
}

Emerg Med J 2004;21:162-164. doi: 10.1136/emj.2002.003244

See end of article for authors' affiliations .....................

Correspondence to: Vicki Leah, Department of Emergency Medicine, Darenth Valley Hospital, Darenth Wood Road Dartford, Kent DA2 8DA, UK;

leah_vicki@hotmail.com

Accepted for publication 18 March 2003
Objectives: To answer the question "In patients presenting with ST elevation acute myocardial infarction (STEMI) and no contraindication to thrombolysis, does the introduction of Tenecteplase reduce door to needle times?"

Methods: Firstly, an observational study was performed to compare the time taken to prepare standard thrombolytic therapy with Tenecteplase. Secondly, door to needle times were compared before and after the introduction of Tenecteplase. The study was powered to be $80 \%$ sure of finding a change of $10 \%$ in the number of patients meeting the national service framework standard of a 30 minute door to needle time. Results: Tenecteplase takes 10.5 minutes less time to prepare than standard treatment ( $p$ value $<0.001$ ). After the introduction of Tenecteplase the percentage of patients receiving thrombolysis in 30 minutes increased from $58 \%$ to $76 \%$ ( $p$ value $<0.01$ )

Conclusion: Tenecteplase is quicker to prepare than standard therapy, resulting in a significant improvement in performance against the national service framework target. l: t has been well established that early intervention with thrombolytic therapy can reduce mortality and morbidity after acute myocardial infarction (AMI). ${ }^{1}$ Thrombolysis within the first hour can save an additional 65 lives per 1000 patients, however a four hour delay will reduce this to 25 lives per 1000 (fig 1).. ${ }^{2}$ The delay between the onset of symptoms and arrival in the emergency department may be many hours, with causes for delay being complex and multifactorial. ${ }^{4}$ Once in hospital further delays can be encountered as a result of delays in assessment, duplication of assessment by a second doctor, or intrahospital transfer if the administration of thrombolysis is confined to a special area outside the emergency department. ${ }^{5}$

Current UK health service performance is not ideal; in 1998 the UKHAS Study ${ }^{6}$ reported that only $2 \%$ of patients received treatment within one hour of symptoms. In an attempt to reduce the mortality and morbidity rates from AMI the national service framework for coronary heart disease ${ }^{7}$ states, in standard six, that "thrombolysis should be given within 60 minutes of calling for help". For acute trusts this translates into a "door to needle" (emergency department arrival to thrombolysis) time of 30 minutes for $75 \%$ of patients presenting to the accident and emergency Department with AMI, which became the national performance standard in April 2002.

Tenecteplase (TNK) is a bolus thrombolytic that was licensed in the UK in 2001. In theory treatment may be given more rapidly, as the drug dissolves quickly without foaming, is packaged in a pre-prepared syringe designed to make it easy to give a weight adjusted dose, and there is no need to use a delivery system, such as a syringe driver.

This study was designed to answer the question "In patients presenting with STEMI (ST elevation acute myocardial infarction) and no contraindications to thrombolysis, does the introduction of Tenecteplase in place of standard therapy reduce door to needle times?"

\section{METHODS}

The study was set in a district general hospital serving a population of 180000 and treating 50000 patients in the emergency department each year. About 160 of these patients are admitted with a diagnosis of AMI, and this population has a median pain to needle time of about 170 minutes. After a period of process mapping a number of delays in the administration of thrombolysis were identified. One seemed to be the time taken to prepare standard thrombolytic therapy. A clinical decision was made to pilot the use of TNK in 40 patients. After this first phase there was a period of six months when TNK was not used, as the business case for the change in clinical practice was prepared. Finally, a change to the use of TNK for all patients took place in January 2002. This series of changes in clinical practice gave the opportunity to study the effects on door to needle times using two prospective observational studies.

During the first phase the preparation time for standard therapy was compared with the preparation time for TNK. Times were recorded from the moment the decision to thrombolyse was made until the time that the infusion pump was prepared for the streptokinase/tPA group, or the syringe was prepared for the TNK group.

Secondly, door to needle times in consecutive patients were compared before and after the introduction of TNK as the only thrombolytic agent used in the hospital. Data were collected as part of the routine system for the myocardial infarction national audit project (MINAP) database. The audit was powered to be $80 \%$ sure of finding a change of $10 \%$ in the NSF 30 minute door to needle time, giving a sample size of 35. Data were therefore collected from all patients who fulfilled the criteria for thrombolysis on arrival in the A\&E department for the four months before and four months after the introduction of TNK. No other known change was made during this time. The grade of medical and nursing staffing of the emergency department did not change, storage of drugs was similar, and there were no other noticeable co-incident developments to emergency cardiac care within the hospital.

Abbreviations: AMI, acute myocardial infarction; STEMI, ST elevation acute myocardial infaction; TNK, Tenecteplase 


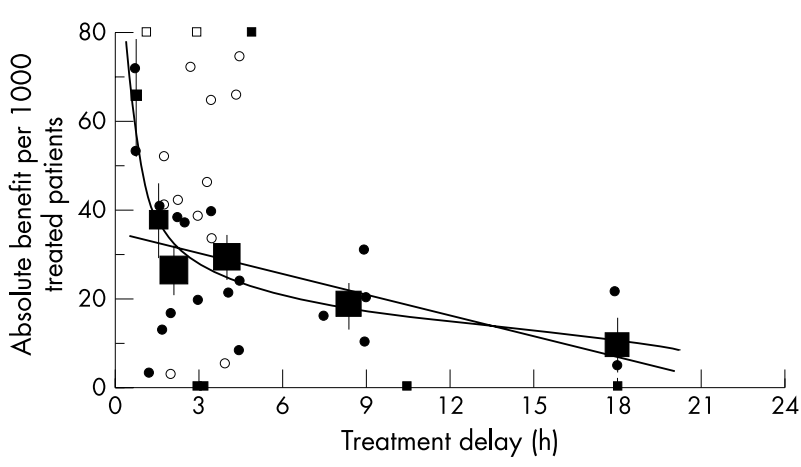

Figure 1 Absolute 35 day motality reduction versus treatment delay. Reprinted with permission from Elsevier Science (Lancet 1996;348: 771-5).

\section{RESULTS}

During the first phase 38 patients received TNK and 30 conventional treatment. The difference in preparation time can clearly be seen (fig 2). In this study TNK took 10.5 minutes less to prepare than standard therapy. The difference is so great and the confidence intervals so small that statistical analysis is not necessary to see that this is a highly significant difference. (Using the Wilcoxon rank sum test the $\mathrm{p}$ value is $<0.001$ ).

In the second study 78 consecutive patients were included, 40 before and 38 after the introduction of TNK. Figure 3 shows the percentage of patients receiving TNK in 10 minute time intervals. More patients receive thrombolysis within the first half hour with TNK, probably indicating that in this group preparation time is an important determinant of door to needle time. While the numbers are quite small it would appear that when there are longer delays, the introduction of TNK had little effect, suggesting that there are other factors affecting the door to needle time in these patients.

From the cumulative data (fig 4) it can be seen that with standard therapy the NSF target of $75 \%$ of patients thrombolysed was met in 40-50 minutes. After the introduction of TNK this target was met in 20-29 minutes. TNK increased the percentage of patients receiving thrombolysis within 30 minutes of presentation from $58 \%$ to $76 \%$. This difference was significant $(\mathrm{p}<0.01)$.

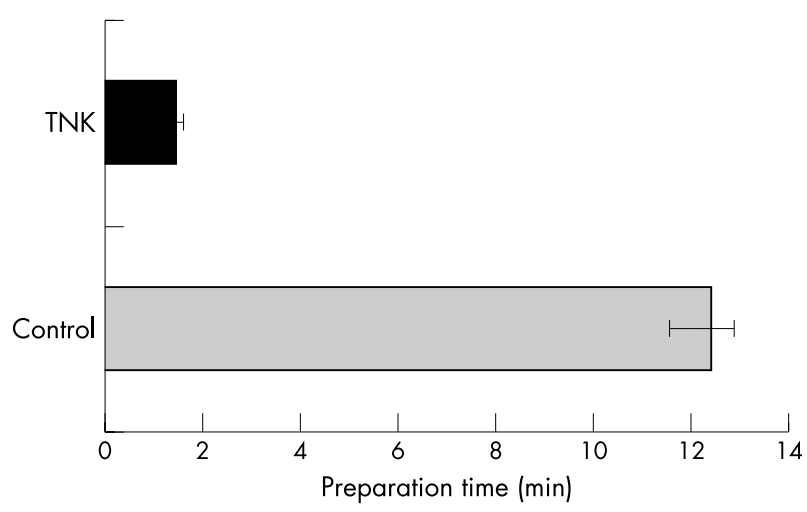

Figure 2 Preparation times.

The study was not powered to examine changes around a 20 minute target, however when the data were evaluated against this level there was a strong trend to benefit from TNK, however significance was not achieved (control 30\%, TNK $42 \%$. $\mathrm{p}=0.07$ ).

\section{DISCUSSION}

While this study has demonstrated a statistically significant reduction in door to needle time with the introduction of $\mathrm{TNK}$, it would be difficult to prove a 10.5 minute reduction resulted in a clinically significant difference terms of patient survival. No cost-benefit analysis was carried out in this study. However, the current pricing of the drug (about $£ 500$ per dose compared with $£ 80$ per dose for streptokinase and $£ 430$ per dose for TPA) would mean an increase in pharmacy costs if TNK was used for all patients.

It could be hypothesised that for patients' presenting late, with several hours from onset of symptoms to presentation, the shape of the Boersma curve (fig 1) means that a 10.5 minute reduction in door to needle time would offer little clinical benefit. Conversely, for patients' presenting early (less than three hours from the onset of symptoms) a 10.5 minute reduction in door to needle time may make a clinically significant difference in terms of survival. So the benefit gained from the introduction of TNK is likely to depend on the pain to needle time of the local population.

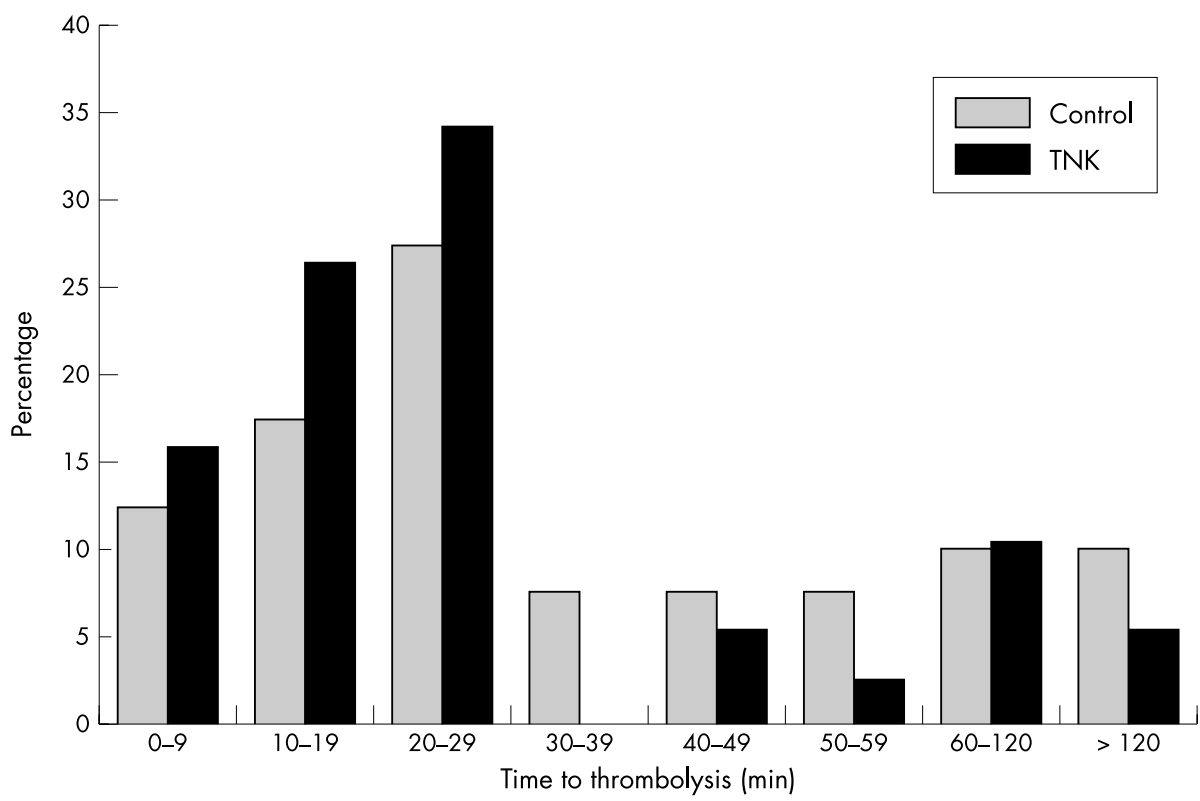

Figure 3 Distribution of door to needle times before and after the introduction of TNK. 


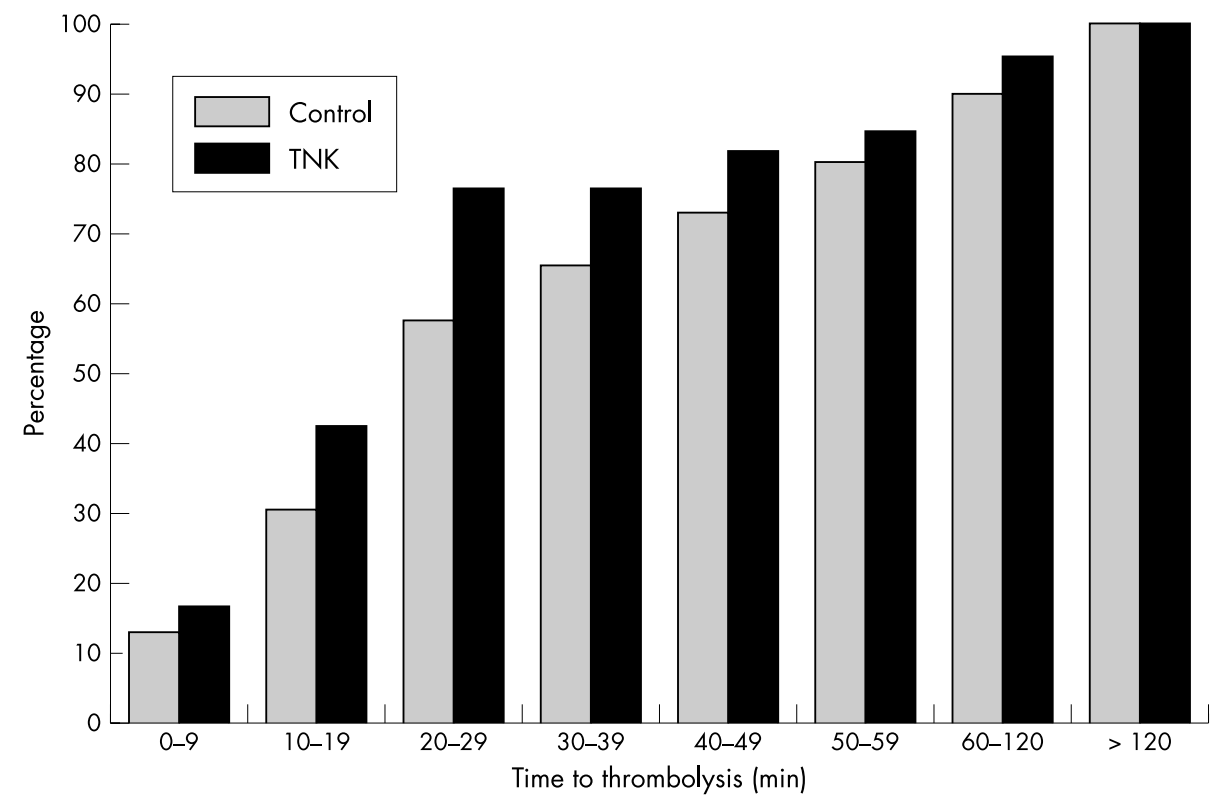

Figure 4 Cumulative door to needle times before and after the introduction of TNK.

Individual trusts will have to make a judgement on whether the increase in cost attributable to the introduction of TNK is justified either in terms of clinical benefit or "administrative benefit" of improving performance against the NSF targets. Our own trust has made the decision to use TNK for: (1) all patients presenting within three hours from the onset of symptoms, (2) all patients with anterior ST elevation, and (3) all patients who have received streptokinase ever before. Inferior ST elevation presenting after three hours from the onset of symptoms in a patient who has not previously received streptokinase will continue to be treated with streptokinase. This protocol means that, from existing MINAP data, we can predict that about $50 \%$ of future patients will receive TNK.

The answer to our initial question seems to be that the introduction of TNK can give a statistically significant improvement in performance against national "door to needle" targets. This may improve a trust's "league table" position, but may not necessarily be cost effective in terms of clinical benefit to the patients.

\section{Authors' affiliations}

V Leah, C Clark, K Doyle, Department of Emergency Medicine, Darent Valley Hospital, Dartford, Kent, UK
T J Coats, Department of Accident and Emergency, St Bartholomew's and the Royal London School of Medicine, Queen Mary's University of London, London, UK

Funding: Boehringer Ingelheim donated without charge the TNK used in this study.

Conflicts of interest: none declared.

\section{REFERENCES}

1 Ohman EM, Harrington RA, Cannon CP, et al. Intravenous thrombolysis in acute myocardial infarction. Chest 2001; 1 19:253-77S.

2 Boersma E, Maas ACP, Deckers JW, et al. Early thrombolytic treatment of acute myocardial infarction: reappraisal of the golden hour. Lancet 1996:348:771-5

3 Fibrinolytic Therapy Trialists' (FTT) Collaborative Group. Indications for fibrinolytic therapy in suspected acute myocardial infarction: collaborative overview of early mortality and major morbidity results from all randomised trials of more than 1000 patients. Lancet 1994:343:311-22.

4 Goldberg RJ, Steg PG, Sadiq I, et al. Extent of, and factors associated with, delay to hospital presentation in patients with acute coronary disease (the GRACE registry. Am J Cardiol 2002;89:791-6.

5 Weston CFM, Penny WJ, Julian DG, on behalf of the British Heart Foundation Working Group. Guidelines for the early management of patients with myocardial infarction. BMJ 1994;308:767-71.

6 The United Kingdom Heart Attack Study (UKHAS) Collaborative Group. Effect of time from onset to coming under care on fatality of patients with acute myocardial infarction: effect of resuscitation and thrombolytic treatment. Heart 1998;80:1 14-20.

7 National Service Framework. Coronary heart disease. March 2000. http:// www.nelh.nhs.uk/nsf/chd/nsf/main/mainreport.htm (accessed 11 Oct 2002). 